Received: 9 January 2017

Accepted: 14 June 2017

Published online: 24 July 2017

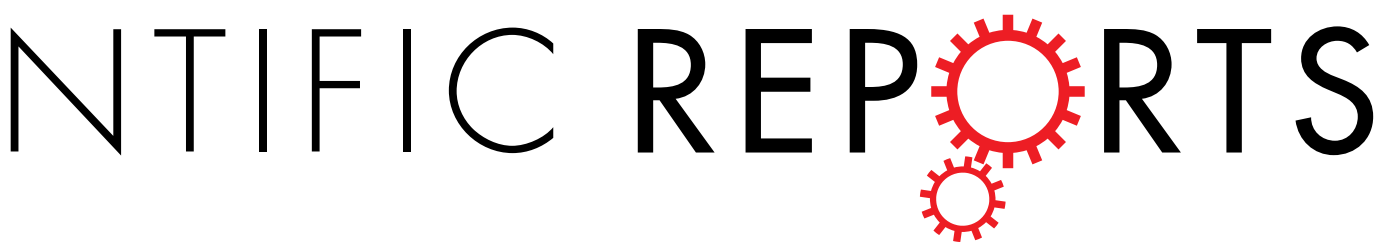

\title{
Validation of asynchronous
} quantitative bone densitometry of the spine: Accuracy, short-term reproducibility, and a comparison with conventional quantitative computed tomography

Ling Wang ${ }^{1}$, Yongbin $\mathrm{Su}^{1}$, Qianqian Wang ${ }^{2}$, Yangyang Duanmu ${ }^{3}$, Minghui Yang ${ }^{4}$, Chen $\mathrm{Yi}^{4}$ \& Xiaoguang Cheng ${ }^{1}$

Asynchronous calibration quantitative computed tomography (OCT) is a new tool that allows the quantification of bone mineral density (BMD) without the use of a calibration phantom during scanning; however, this tool is not fully validated for clinical use. We used the European spine phantom (ESP) with repositioning during scanning and assessed the accuracy and short-term reproducibility of asynchronous $\mathrm{QCT}$. Intra-scanner and intra-observer precision were each calculated as the root mean square of the standard deviation (RMSSD) and the coefficient of variation (CV-RMSSD). We also compared asynchronous and conventional QCT results in 50 clinical subjects. The accuracy of asynchronous QCT for three ESP vertebrae ranged from 1.4-6.7\%, whereas intra-scanner precision for these vertebrae ranged from $0.53-0.91 \mathrm{mg} / \mathrm{cc}$. Asynchronous $\mathrm{QCT}$ was most precise for a trabecular BMD of $100 \mathrm{mg} / \mathrm{cc}(\mathrm{CV}-\mathrm{RMSSD}=0.2 \%)$. For intra-observer variability, overall precision error was smaller than $3 \%$. In clinical subjects there was excellent agreement between the two calibration methods with correlation coefficients ranging from 0.96-0.99. A Bland-Altman analysis demonstrated that methodological differences depended on the magnitude of the BMD variable. Our findings indicate that the asynchronous QCT has good accuracy and precision for assessing trabecular BMD in the spine.

Bone mineral density is a surrogate indicator of bone strength that plays an important role in the management of osteoporosis and related fractures ${ }^{1,2}$. Different from areal bone mineral density computed by dual-energy X-ray absorptiometry (DXA), bone mineral density (BMD) derived from quantitative computed tomography (QCT) is a volumetric measure of the vertebral trabecular bone. Given the high turnover rate of trabecular bone compared to cortical bone, BMD calculated from QCT offers substantially higher sensitivity. Yet, radiation doses associated with CT limit the application of QCT in osteoporosis screening.

Recently, asynchronous calibration QCT was described as a new tool for quantifying BMD ${ }^{3}$. Asynchronous calibration means that a calibration phantom is not necessary during QCT scanning. Rather, asynchronous QCT utilizes phantom data obtained separately from CT scans to calibrate data in Hounsfield units for the measurement of BMD. This approach is convenient for the assessment of BMD during routine abdominal and/or lung CT scans, and for the identification of patients who have an increased risk of fracture with diagnostic CT scanning. The 2015 International Society for Clinical Densitometry (ISCD) official position states that the in-scan

${ }^{1}$ Department of Radiology, Peking University Fourth School of Clinical Medicine, Beijing Jishuitan Hospital, Xicheng District, Beijing, China. ${ }^{2}$ Department of Epidemiology, Peking University Fourth School of Clinical Medicine, Beijing Jishuitan Hospital, Xicheng District, Beijing, China. ${ }^{3}$ Department of Radiology, Forth Affiliated Hospital of Anhui Medical University, Anhui, China. ${ }^{4}$ Department of Traumatology and Orthopedic Surgery, Peking University Fourth School of Clinical Medicine, Beijing Jishuitan Hospital, Xicheng District, Beijing, China. Correspondence and requests for materials should be addressed to X.C. (email: xiao65@263.net) 


\begin{tabular}{|l|l|l|}
\hline & European spine phantom & Patient spine \\
\hline Scanner type & 80 -slice Toshiba Aquilion & 16 -slice Toshiba Aquilion \\
\hline Voltage $(\mathrm{kV})$ & 120 & 120 \\
\hline Exposure $(\mathrm{mAs})$ & 100 & Auto exposure \\
\hline Reconstruction kernel & Standard & Standard \\
\hline DFOV $(\mathrm{mm})$ & 400 & 400 \\
\hline Slice thickness $(\mathrm{mm})$ & 1.0 & 1.0 \\
\hline Table height $(\mathrm{cm})$ & 120 & 90 \\
\hline
\end{tabular}

Table 1. Computed tomography (CT) scan parameters for phantom and patient image acquisition. DFOV, display field of view.

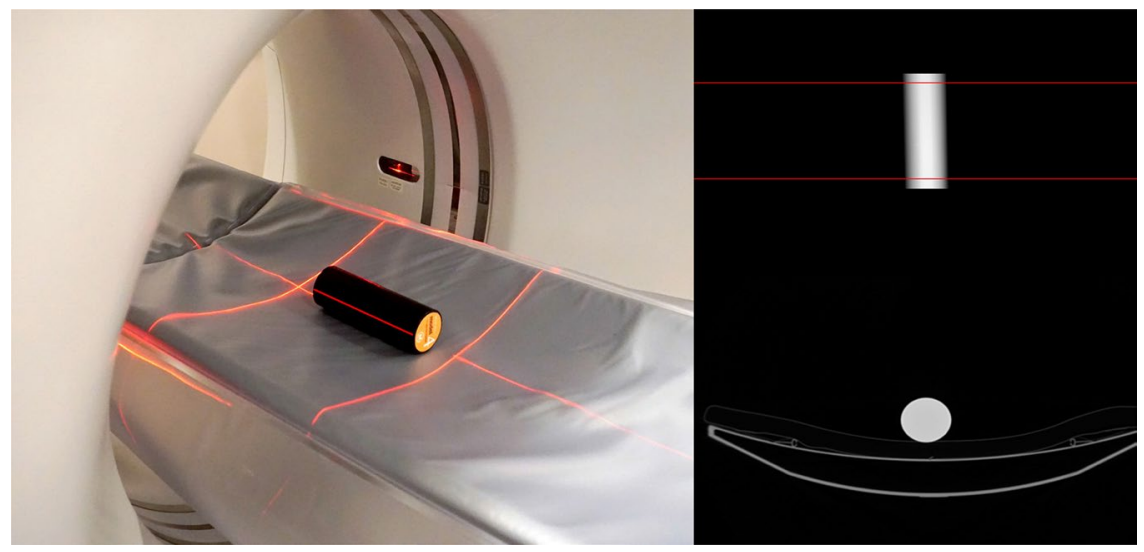

Figure 1. Quality assurance (QA) for asynchronous quantitative computed tomography using the Model 4 asynchronous QA phantom.

calibration phantom for density-based QCT measurement can be replaced with asynchronous calibration if scanner stability is maintained ${ }^{4,5}$. Opportunistic screening enabled by the use of asynchronous calibration may therefore improve the current understanding of bone health status and decrease the number of undiagnosed or overlooked cases of osteoporosis.

Few studies to date have compared conventional QCT, asynchronous QCT, and DXA ${ }^{3,6-8}$. Moreover, asynchronous QCT has not yet been fully validated for the clinical measurement of spinal BMD. Although an early study by Brown et al. reported the precision of asynchronous QCT using the Mindways QA phantom, this study did not perform an accuracy assessment using the European spine phantom (ESP), which is a standard evaluation tool for bone densitometry. Thus, we performed 10 ESP scans with repositioning in order to assess the accuracy and short-term reproducibility of asynchronous quantitative bone densitometry, and to compare spine BMD results of asynchronous and conventional QCT in 50 clinical subjects.

\section{Methods}

Evaluation of accuracy and short-term reproducibility with ESP. The ESP (QRM, Erlangen, Germany) was recommended by the International DXA Standardization Committee as a possible standard for use in DXA as well as QCT 9 . The ESP consists of three simulated vertebrae that are designed to give trabecular density values of 50,100 , and $200 \mathrm{mg} / \mathrm{cm}^{3}$ of hydroxyapatite, respectively. To simulate slight differences seen on daily quality control (QC) charts due to repositioning of the phantom, we performed each phantom scan with repositioning of the ESP. This method was recommended in the 2005 ISCD Official Position and reported to improve the estimate of the mean measure by a factor of nearly three ${ }^{10}$. Briefly, the ESP was placed on the scanner table on top of the table pad and aligned along the long axis of the table. CT scanning parameters were summarized in Table 1. Images were transferred to a QCT workstation and analysed using the 3D spine function version 5.10 of Mindways QCT pro software (Mindways Software Inc., Austin, TX, USA). This novel version includes a conventional QCT module and an asynchronous calibration module. For asynchronous QCT measurements, a new Model 4 asynchronous calibration phantom (Mindways Software Inc.) (Fig. 1) was scanned for quality assurance (QA) calibration. A Model 3 conventional calibration phantom (Mindways Software Inc.) was used to validate QA calibration for conventional QCT analysis. To evaluate short-term intra-scanner precision, a tenscan series was performed in duplicate over two sessions, with one month between each scanning session. For the evaluation and comparison of asynchronous and conventional QCT, a Toshiba Aquilion 80-slices CT scanner (Toshiba Medical Systems Corp., Tokyo, Japan) was used to scan the ESP (no. 145) in the presence and absence of a Mindways calibration phantom, ten times each. Each group of ten scans was analysed individually and the average of each parameter was used to compute accuracy. To further compare of the effect of inter-scanner differences, 


\begin{tabular}{|l|l|l|l|l|l|l|l|l|l|l|}
\hline & \multicolumn{3}{|l|}{ Accuracies of asynchronous and conventional QCT using 80-slice CT scanner } & \multicolumn{2}{l|}{ Inter-scanner differences } \\
\hline Site & $\begin{array}{l}\text { ESP BMD } \\
\left(\mathrm{mg} / \mathrm{cm}^{3}\right)\end{array}$ & $\begin{array}{l}\text { Conv. values } \\
\left(\mathrm{mg} / \mathrm{cm}^{3}\right)\end{array}$ & RE & $\begin{array}{l}\text { Asyc. values } \\
\left(\mathrm{mg} / \mathrm{cm}^{3}\right)\end{array}$ & RE & $\begin{array}{l}\text { Conv. Asyc. } \\
\left(\mathrm{mg} / \mathrm{cm}^{3}\right)\end{array}$ & P-value & $\begin{array}{l}\text { Conv. values on } \\
16-\text {-slice scanner } \\
\left(\mathrm{mg} / \mathrm{cm}^{3}\right)\end{array}$ & Diff. $\left(\mathrm{mg} / \mathrm{cm}^{3}\right)$ & P-value \\
\hline L1 & 50 & $52.94 \pm 0.99$ & $5.9 \%$ & $51.89 \pm 0.53$ & $3.8 \%$ & 1.05 & $<0.0001$ & $52.14 \pm 1.45$ & 0.80 & 0.66 \\
\hline L2 & 100 & $104.18 \pm 0.85$ & $4.2 \%$ & $101.40 \pm 0.61$ & $1.4 \%$ & 2.78 & $<0.0001$ & $105.00 \pm 0.37$ & -0.82 \\
\hline L3 & 200 & $207.40 \pm 0.97$ & $3.7 \%$ & $186.70 \pm 0.53$ & $6.7 \%$ & 20.71 & 0.0029 & $204.85 \pm 1.35$ & 2.55 & 0.37 \\
\hline
\end{tabular}

Table 2. The accuracies of asynchronous and conventional quantitative computed tomography (QCT) and comparisons of inter-scanner differences. Asyc., asynchronous QCT method; BMD, bone mineral density; Conv., conventional QCT; ESP, European spine phantom; RE, Relative error; Diff. difference defined as 80-slice scanner - 16-slice scanner. Data represent the mean \pm standard deviation.

The ESP was also scanned in 16-slice CT scanner for ten times with a calibration phantom. Based on the BMD measured using conventional QCT on each scanner in the different ESP regions, the interchangeability of the two CT systems was showed. Scan parameters of the two CT scanners were summarized on Table 1.

Subjects and clinical data. Fifty subjects ( 25 female subjects and 25 male subjects) were retrospectively enrolled in August 2016, one week before the date of Model4 Quality Assurance Scanning, from the emergency room radiology department of Beijing Jishuitan Hospital. In our emergency room radiology department, there are 10 to 20 clinical spine QCT scans on each day. For inclusion, eligible patients had to have conventional spine QCT BMD examinations within one week to avoid uncertainty regarding calibration stability in retrospective measurements. The exclusion criteria included fixation of the spine affecting image quality and more than two vertebrae compressive fractures. The 3D spine function version 5.10 of Mindways QCT pro software (Mindways Software Inc., Austin, TX, USA) was used to perform L1-L3 BMD analysis of all 50 cases, once with the conventional QCT module and once with the asynchronous calibration module. To further assess intra-observer precision, all 50 cases were analysed with the asynchronous QCT module two times by one radiologist, with one week between each analysis. This study was approved by the Ethics Committee of Beijing Jishuitan Hospital, Xicheng District, Beijing, China, and all methods were performed in accordance with the approved guidelines, and all the subjects signed informed consents.

Image acquisition. For ESP, QCT scans were acquired using a Toshiba Aquilion 80-slice CT scanner (Toshiba Medical Systems Corp., Tokyo, Japan) with and without a calibration phantom (Mindways Software Inc., Austin, TX, USA). For clinical assessments, spine QCT scans were acquired using a Toshiba Aquilion 16-slice CT scanner (Toshiba Medical Systems Corp., Tokyo, Japan) with a calibration phantom (Mindways Software Inc., Austin, TX, USA). As the clinical scans were retrospectively from patients who aimed to have conventional QCT examinations, in our department the clinical QCT scans were all performed by 16-slice CT scanner in the emergency room radiology department. The reason for performing ESP scans on 80-slice CT scanner is that we want to investigate the intra-scanner precision on another scanner to show the equivalence. Scan parameters for both CT scanners were as follows: $120 \mathrm{kVp}, 125 \mathrm{mAs}, 1-\mathrm{mm}$ slice thickness, 50 -cm scan field of view, and a matrix size of $512 \times 512$ in spiral and standard reconstructions.

Statistics analysis. The accuracies of conventional and asynchronous QCT for ESP scans were assessed by calculating the average of percentages of difference between measured values and expected values given by the manufacturer. Mann-Whitney U tests were used to compare values acquired using the two methods and to compare the interchangeability of the two CT scanners systems. A linear regression analysis was used to compare the density values acquired using the two methods, and a Bland-Altman analysis was conducted to assess differences between the conventional and asynchronous QCT measurements. As per the ISCD recommendation, short-term precision (intra-observer and intra-scanner precision) was calculated in terms of RMSSD and CV-RMSSD values.

Data Availability. The datasets generated during and/or analyzed during the current study are available from the corresponding author on reasonable request.

\section{Results}

Accuracy. The results of the accuracy analysis based on 10 ESP scans with repositioning are summarized in Table 2. The accuracies of conventional and asynchronous QCT were different, ranging from 3.7-5.9\% for conventional QCT and from 1.4-6.7\% for asynchronous QCT. Asynchronous QCT had higher accuracy than conventional QCT for measuring trabecular BMD in 50 and $100 \mathrm{mg} / \mathrm{cc}$ ESP (L1 and L2, respectively) vertebrae, whereas conventional QCT showed better accuracy for measuring the $200 \mathrm{mg} / \mathrm{cc}$ ESP L3 vertebra. Conventional QCT tended to overestimate ESP trabecular bone, whereas asynchronous QCT underestimated the BMD of the ESP L3 vertebra. The mean trabecular volumetric BMD (vBMD) for all three ESP vertebrae using conventional QCT was significantly higher than that obtained using asynchronous QCT. Figure 2 shows the results of ten scans performed with asynchronous QCT and conventional QCT; consistency was excellent for both methods.

Intra-scanner short-term reproducibility. The intra-scanner precision of asynchronous QCT for vBMD across all three vertebrae ranged from $0.53-0.91 \mathrm{mg} / \mathrm{cc}$. Asynchronous QCT was most precise (in terms of the 


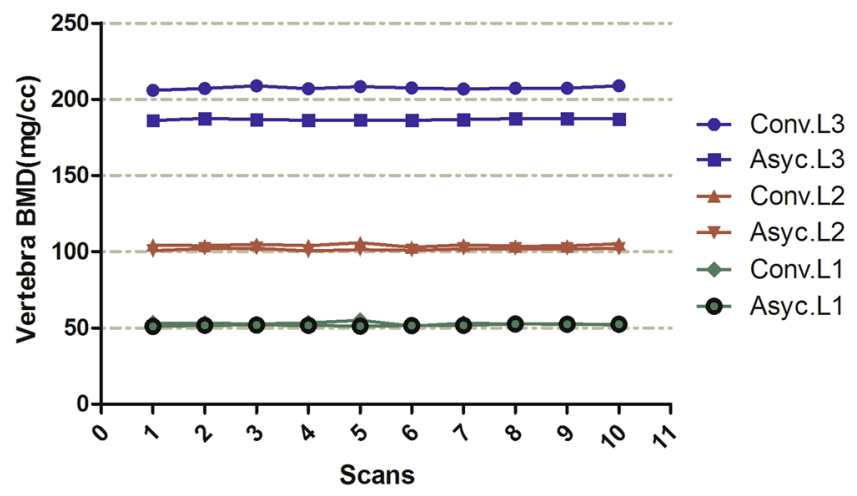

Figure 2. Ten-scan results of asynchronous and conventional quantitative computed tomography (QCT) for ESP study. Asyc., asynchronous QCT method; Conv., conventional QCT method.

\begin{tabular}{|l|l|l|l|}
\hline & $\begin{array}{l}\text { Male subjects } \\
(\mathbf{n = 2 5})\end{array}$ & $\begin{array}{l}\text { Female subjects } \\
(\mathbf{n = 2 5})\end{array}$ & $\begin{array}{l}\text { Total subjects } \\
(\mathbf{n = 5 0})\end{array}$ \\
\hline Age (years) & $62 \pm 11$ & $62 \pm 8$ & $62 \pm 9$ \\
\hline Weight $(\mathrm{kg})$ & $76.1 \pm 7.0$ & $65.1 \pm 9.1$ & $70.7 \pm 9.8$ \\
\hline Height $(\mathrm{cm})$ & $170.6 \pm 5.7$ & $161.0 \pm 6.0$ & $165.9 \pm 7.9$ \\
\hline BMI $\left(\mathrm{kg} / \mathrm{m}^{2}\right)$ & $26.2 \pm 1.9$ & $25.1 \pm 3.3$ & $25.6 \pm 2.7$ \\
\hline Osteoporosis & $10 / 25$ & $12 / 25$ & $22 / 50$ \\
\hline
\end{tabular}

Table 3. Characteristics of clinical subjects with QCT scans acquired using a 16-slice CT scanner. BMI, body mass index. Data represent the mean \pm standard deviation.

root mean square of the standard deviation [RMSSD]) for the $100 \mathrm{mg} / \mathrm{cc}$ ESP L2 vertebra with a coefficient of variance $(\mathrm{CV})$-RMSSD value of $0.2 \%$.

Clinical study. Table 3 shows that among 25 male subjects and 25 female subjects, the mean age was $62 \pm 9$ years (range, $33-85$ years), the mean height was $165.9 \pm 7.9 \mathrm{~cm}$ (range, $150.0-181.0 \mathrm{~cm}$ ), the mean weight was $70.7 \pm 9.8 \mathrm{~kg}$ (range, $50.0-89.5 \mathrm{~kg}$ ), and the mean BMI was $25.6 \pm 2.7 \mathrm{~kg} / \mathrm{m}^{2}$ (range, $20.4-32.8 \mathrm{~kg} / \mathrm{m}^{2}$ ). Of the 50 subjects, twelve female subjects and 10 male subjects had osteoporosis, and two patients had other vertebrae compressive fractures.

Relationships between conventional and asynchronous QCT scanning results. Figure 3 shows correlations between the results of conventional and asynchronous QCT for each vertebra (L1-L3) and the average of all 3 vertebrae of the clinical subjects. The analysis demonstrated excellent agreement between the two methods, with correlation coefficients $\left(\mathrm{R}^{2}\right)$ ranging from 0.96-0.99. A Bland-Altman analysis (small upper panel inserts in Fig. 3) showed differences between conventional and asynchronous QCT for different vertebrae with respect to a linear correlation. Methodological differences (expressed as absolute differences) depended on the magnitude of the BMD variable.

Intra-observer variability. Table 4 summarizes the RMMS and CV-RMSS values for repeated measures with the asynchronous calibration method. The intra-observer precision for asynchronous QCT measured across all three ESP vertebrae ranged from $2.68-3.80 \mathrm{mg} / \mathrm{cc}$. Overall precision error for BMD was smaller than $3 \%$, consistent with the known precision of QCT technology.

\section{Discussion}

In this study, we determined the accuracy and intra-operator precision of asynchronous QCT using an ESP vertebrae dataset and data retrospectively collected from clinical subjects. In both datasets, the precision of BMD measurement was around 3\%. Our results suggest that vertebral trabecular BMD measurements can be conveniently obtained by asynchronous QCT with good accuracy and precision.

The results of an analysis based on ten ESP scans demonstrated that asynchronous QCT had excellent accuracy for measuring trabecular BMD and agreed well with conventional QCT. Asynchronous QCT had slightly higher accuracy than conventional QCT for measuring trabecular BMD in 50 and $100 \mathrm{mg} / \mathrm{cc}$ ESP vertebrae; however, the differences between the two methods for 50 and $100 \mathrm{mg} / \mathrm{cc}$ vertebrae were relatively small $(1.05 \mathrm{mg} /$ $\mathrm{cc}$ and $2.78 \mathrm{mg} / \mathrm{cc}$, respectively). In contrast, conventional QCT was more accurate for measuring the $200 \mathrm{mg} / \mathrm{cc}$ ESP vertebra, suggesting that the asynchronous QCT method may underestimate the BMD of high-density bone. While traditional QCT utilizes a calibration equation obtained from each individual slice, asynchronous QCT uses the same calibration equation for all data. This may result in lower variability in high-BMD bone for asynchronously calibrated results. Inconsistent with our finding, Brown et al. showed excellent agreement between conventional and asynchronous QCT using high-BMD Mindways QA phantom scan data ${ }^{3}$. The reason for this 

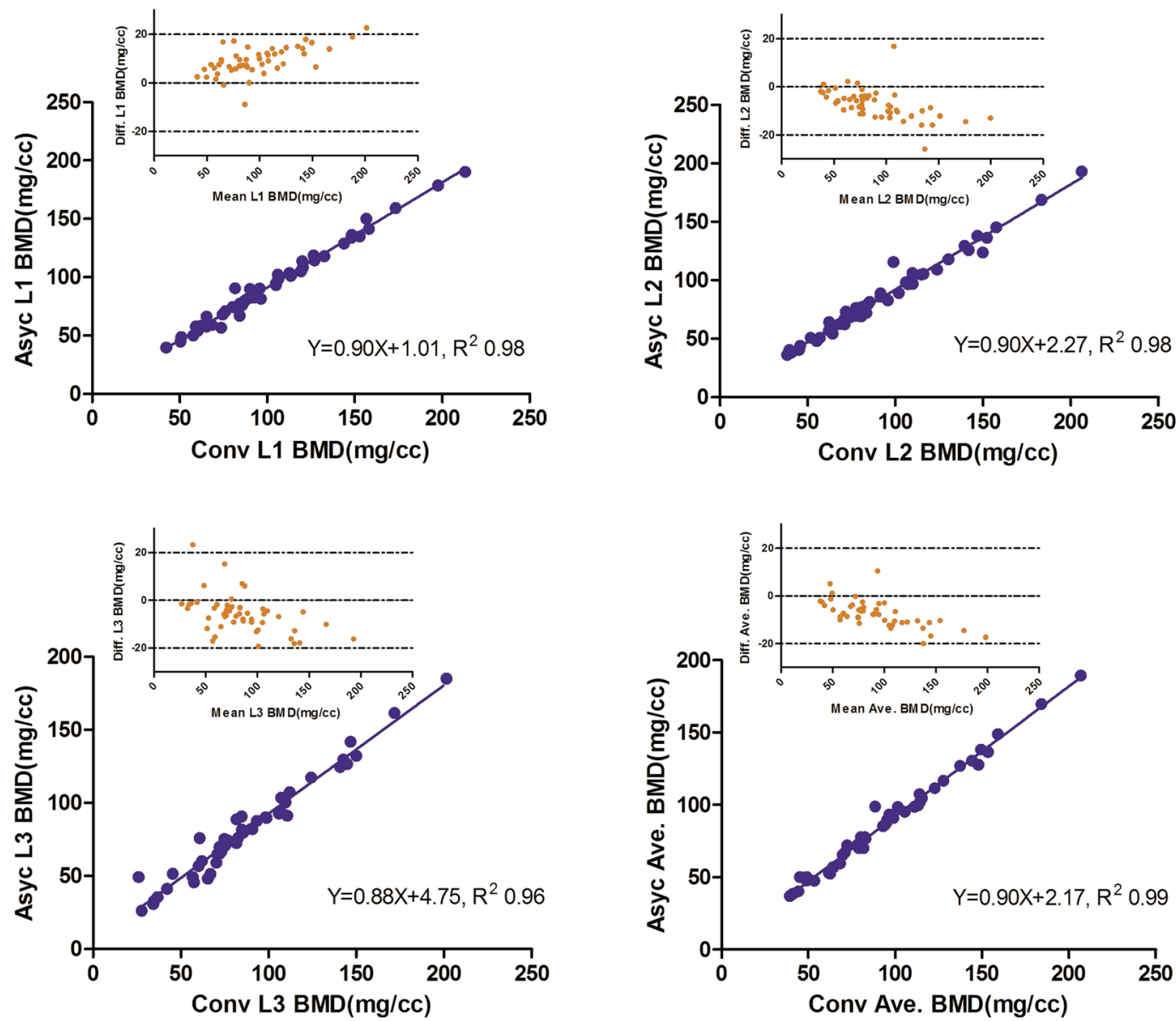

Figure 3. Correlation scatter plots for conventional and asynchronous quantitative computed tomography (QCT) outcomes of clinical subjects and Bland-Altman plots (small upper panel inserts) for conventional and asynchronous QCT BMD measurements of clinical subjects. Small upper inserts: Y-axis (Diff) is defined as (Asyc. -Conv.); $\mathrm{X}$-axis is defined as the mean value of (Asyc. +Conv.).

\begin{tabular}{|l|l|l|l|l|l|l|l|l|l|}
\hline \multicolumn{9}{|l|}{ Intra-observer variability } & \multicolumn{1}{l|}{ Intra-scanner variability } \\
\hline $\begin{array}{l}\text { Subjects } \\
(\mathrm{n}=50)\end{array}$ & Obs. 1 & Obs. 2 & RMSSD & CV-RMSSD & $\begin{array}{l}\text { ESP scans } \\
(\mathrm{n}=10)\end{array}$ & Scan 1 & Scan 2 & RMSSD & CV-RMSSD \\
\hline L1 vBMD & $93.04 \pm 34.58$ & $94.02 \pm 35.14$ & 3.12 & 2.5 & L1 vBMD & $51.89 \pm 0.53$ & $51.79 \pm 0.20$ & 0.56 & 0.7 \\
\hline L2 vBMD & $86.76 \pm 34.01$ & $87.64 \pm 34.43$ & 3.73 & 2.6 & L2 vBMD & $101.40 \pm 0.61$ & $101.67 \pm 0.40$ & 0.91 & 0.6 \\
\hline L3 vBMD & $81.44 \pm 33.42$ & $81.39 \pm 33.51$ & 2.68 & 2.2 & L3 vBMD & $186.70 \pm 0.53$ & $186.55 \pm 0.46$ & 0.53 & 0.2 \\
\hline
\end{tabular}

Table 4. Intra-observer and intra-scanner reproducibility of asynchronous quantitative computed tomography (QCT). CV, coefficient of variation; Obs., observation; RRMSD, root mean square of the standard deviation; vBMD, volumetric bone mineral density. Data represent the mean \pm standard deviation.

discrepancy is unclear; however, one explanation may be related to the fact that peak histogram values used in the calibration procedure depend on noise and thus exposure settings, the reconstruction kernel, and slice thickness ${ }^{5}$.

True in vivo precision measurements were obtained by measuring subjects twice at a one-month interval with repositioning. Repositioning has a lower impact on precision in three-dimensional QCT than in traditional two-dimensional slice-based imaging, where the location of the slice relative to the vertebral body is determined at the time of acquisition using the scout view taken before the actual CT scan. In an earlier study, Brown et al. ${ }^{3}$ investigated inter-observer variability for the asynchronous method using data from 43 patients aged $63.8 \pm 8.6$ years and reported RMSSD and CV-RMSSD values of $4.34 \mathrm{mg} / \mathrm{cc}$ and $3.67 \%$, respectively; bias was not considered to be clinically important in the context of osteoporosis screening. In our study, we assessed the intra-observer reproducibility for asynchronous QCT and calculated RMSSD values of 3.12, 3.73, and $2.68 \mathrm{mg} / \mathrm{cc}$ and CV-RMSSD values of 2.5\%, 2.6\%, and 2.2\% for the ESP L1-L3 vertebrae, respectively. Inter-observer reproducibility for asynchronous QCT reported by Brown et al. is therefore similar to the calculated intra-observer variability in our study. 
In one phantomless QCT BMD study, inter-observer variability was $3.1 \mathrm{mg} / \mathrm{cc}$ and CV-RMSSD was $4.0 \%{ }^{11}$, which are high compared to the results of our study. Although previous reports have indicated that non-calibrated Hounsfield unit values from CT scanners may be used for the opportunistic screening of low bone mass ${ }^{11-13}$, the use of a phantom calibration standard guarantees that the derived BMD computations will be consistent across CT scanners from different manufacturers and consistent across different scanning X-ray energy levels.

Lastly, we found that asynchronous and synchronous QCT results were highly correlated; regression lines for each method were not significantly different. Accordingly, it appears that bias (expressed as the absolute difference between method results) depends on the magnitude of the BMD variable. Different from conventional QCT utilizing a calibration equation obtained from each individual slice, the asynchronous QCT uses the same calibration equation for all slices and could have such calibration before or after CT images acquisition. The different calibration methods might be one of important causes of the bias. Further, there might be a phantom-induced bias between asynchronous and conventional QCT. Brown et al. had investigated the phantom-induced bias on clinical individuals and found that the bias induced by the presence of the phantom was $2.3 \mathrm{mg} / \mathrm{cm} 3$ when asynchronous calibration was applied ${ }^{3}$.

The present study had several limitations that most notably affected our precision assessments. First, due to concerns about radiation doses, it was not appropriate to scan patients twice in one session, so we performed ESP scans to investigate intra-scanner bias; however, this approach does not meet conservative recommendations ( $>27$ degree of freedom, DOF) and may have underestimated precision error ${ }^{14}$. Second, we did not analyse the long-term precision of asynchronous QCT; this should be investigated in future studies. Lastly, the BMD of volunteers recruited in precision studies is often normal. In this study, we selected patients from a population with low-to-normal BMD. Given that BMD typically decreases with age, precision errors are typically larger in elderly individuals ${ }^{15}$. Additionally, precision errors are typically estimated by scanning a subject twice on the same day with repositioning in between scans and all scans performed by the same operator. Instead, we report precision using repeat scans taken at a one-month interval. We believe that these aspects of our study provide a more accurate reflection of the real precision of asynchronous QCT measurement. Further studies are necessary to confirm and extend our results.

In conclusion, we demonstrate that asynchronous QCT could be used for spine BMD screening based on the results of accuracy assessment for volumetric trabecular BMD in the spine and good short-term precision; however, even when the asynchronous and synchronous methods are highly correlated, the presence of bias was observed.

\section{References}

1. Cummings, S. R. \& M., L. Epidemiology and outcomes of osteoporotic fractures. Lancet 359, 1761-1767 (2002).

2. Engelke, K. Assessment of bone quality and strength with new technologies. Current opinion in endocrinology, diabetes, and obesity 19, 474-482, doi:10.1097/MED.0b013e32835a2609 (2012).

3. Brown, J. K. et al. Asynchronously Calibrated Quantitative Bone Densitometry. J Clin Densitom, doi:10.1016/j.jocd.2015.11.001 (2016).

4. Shepherd, J. A., Schousboe, J. T., Broy, S. B., Engelke, K. \& Leslie, W. D. Executive Summary of the 2015 ISCD Position Development Conference on Advanced Measures From DXA and QCT: Fracture Prediction Beyond BMD. J Clin Densitom 18, 274-286, doi:10.1016/j.jocd.2015.06.013 (2015).

5. Engelke, K. et al. Clinical Use of Quantitative Computed Tomography-Based Advanced Techniques in the Management of Osteoporosis in Adults: the 2015 ISCD Official Positions-Part III. J Clin Densitom 18, 393-407, doi:10.1016/j.jocd.2015.06.010 (2015).

6. Pickhardt, P. J., Bodeen, G., Brett, A., Brown, J. K. \& Binkley, N. Comparison of Femoral Neck BMD Evaluation Obtained Using Lunar DXA and QCT With Asynchronous Calibration From CT Colonography. Journal of Clinical Densitometry 18, 5-12, doi:10.1016/j.jocd.2014.03.002 (2015).

7. Ziemlewicz, T. J. et al. Direct Comparison of Unenhanced and Contrast-Enhanced CT for Opportunistic Proximal Femur Bone Mineral Density Measurement: Implications for Osteoporosis Screening. AJR. American journal of roentgenology 206, 694-698, doi:10.2214/AJR.15.15128 (2016).

8. Ziemlewicz, T. J. et al. Opportunistic Quantitative CT Bone Mineral Density Measurement at the Proximal Femur Using Routine Contrast-Enhanced Scans: Direct Comparison with DXA in 355 Adults. J Bone Miner Res, doi:10.1002/jbmr.2856 (2016).

9. Pearson, J. et al. European semi-anthropomorphic spine phantom for the calibration of bone densitometers: assessment of precision, stability and accuracy. The European Quantitation of Osteoporosis Study Group. Osteoporosis international: a journal established as result of cooperation between the European Foundation for Osteoporosis and the National Osteoporosis Foundation of the USA 5, 174-184 (1995)

10. Shepherd, J. A. et al. Cross-calibration and minimum precision standards for dual-energy X-ray absorptiometry: the 2005 ISCD Official Positions. J Clin Densitom 9, 31-36, doi:10.1016/j.jocd.2006.05.005 (2006).

11. Mueller, D. K. et al. Phantom-less QCT BMD system as screening tool for osteoporosis without additional radiation. European journal of radiology 79, 375-381, doi:10.1016/j.ejrad.2010.02.008 (2011).

12. Pickhardt, P. J. et al. Opportunistic screening for osteoporosis using abdominal computed tomography scans obtained for other indications. Annals of internal medicine 158, 588-595, doi:10.7326/0003-4819-158-8-201304160-00003 (2013).

13. Kaesmacher, J., Liebl, H., Baum, T. \& Kirschke, J. S. Bone Mineral Density Estimations From Routine Multidetector Computed Tomography: A Comparative Study of Contrast and Calibration Effects. Journal of computer assisted tomography, doi:10.1097/ RCT.0000000000000518 (2016).

14. Gluer, C. C. et al. Accurate assessment of precision errors: how to measure the reproducibility of bone densitometry techniques. Osteoporosis international: a journal established as result of cooperation between the European Foundation for Osteoporosis and the National Osteoporosis Foundation of the USA 5, 262-270 (1995).

15. Berntsen, G. K., Fonnebo, V., Tollan, A., Sogaard, A. J. \& Magnus, J. H. Forearm bone mineral density by age in 7,620 men and women: the Tromso study, a population-based study. Am J Epidemiol 153, 465-473 (2001).

\section{Acknowledgements}

This work was supported by grants from National Natural Science foundation of China (Grant no: 81071131;81401407), the Beijing Bureauof Health 215 program (Grant no:2013-3-033; 2009-2-03), Capital Characteristic Clinic Project (Grant no:Z141107002514072), Beijing Talents Fund(Grant no: 2015000021467G177). 


\section{Author Contributions}

L.W. is the primary author and contributed to the manuscript in the following ways: conception and design, patient selection, analysis and interpretation of data, manuscript drafting and revision. The following authors contributed as described: Y.S. (participated in the design of the study, analyzed data, interpreted results, and revised the manuscript), Q.W. (analyzed data, interpreted results,), Y.D. (performed CT Scans, patient selection and revision), M.Y. and C.Y. (patient selection), and X.C. (study supervisor, conception and design, data analysis and interpretation, manuscript revision).

\section{Additional Information}

Competing Interests: The authors declare that they have no competing interests.

Publisher's note: Springer Nature remains neutral with regard to jurisdictional claims in published maps and institutional affiliations.

(c) (i) Open Access This article is licensed under a Creative Commons Attribution 4.0 International License, which permits use, sharing, adaptation, distribution and reproduction in any medium or format, as long as you give appropriate credit to the original author(s) and the source, provide a link to the Creative Commons license, and indicate if changes were made. The images or other third party material in this article are included in the article's Creative Commons license, unless indicated otherwise in a credit line to the material. If material is not included in the article's Creative Commons license and your intended use is not permitted by statutory regulation or exceeds the permitted use, you will need to obtain permission directly from the copyright holder. To view a copy of this license, visit http://creativecommons.org/licenses/by/4.0/.

(C) The Author(s) 2017 